\title{
Data Science for Characterizing Breast Cancer
}

\author{
Wang Lingxiao* \\ Beijing University of Technology, BDIC, China Beijing, 100124
}

\begin{abstract}
*With the development of society, data science such as machine learning, data visualization and other issues have expanded to various scientific fields and affect our lives from various aspects. In this article, we will analyze the data of breast cancer characteristics from the University Medical Centre, Institute of Oncology, Ljubljana, Yugoslavia, categorize the characteristics of breast cancer by using data visualization method, and learn and predict the whole data by random forest model to obtain the most influential factors for the development and recurrence of breast cancer. It will have a positive impact on the prevention of breast cancer in the future. Also the reliability of the algorithm need to prove.
\end{abstract}

\section{CCS CONCEPTS}

- Computing methodologies $\rightarrow$ Machine learning; Machine learning approaches.

\section{KEYWORDS}

Machine learning, breast cancer, data science, Random forest

\section{ACM Reference Format:}

Wang Lingxiao. 2021. Data Science for Characterizing Breast Cancer. In 2021 3rd International Conference on Intelligent Medicine and Image Processing (IMIP '21), April 23-26, 2021, Tianjin, China. ACM, New York, NY, USA, 5 pages. https://doi.org/10.1145/3468945.3468965

\section{INTRODUCTION}

Breast cancer, one of the most prevalent cancers today, has become a focus of attention for people around the world, with $12 \%$ of women in the United States being diagnosed with breast cancer during their lifetimes and more than 250,000 new breast cancer cases diagnosed in the United States in 2017 [2] and 1 million new cases worldwide each year, making breast cancer the most common malignancy in women, accounting for $18 \%$ of all female cancers. In the UK, agestandardised morbidity and mortality rates are among the highest in the world, with an incidence rate of nearly 2 cases per 1000 women per year in women aged 50 years. The disease is the most common cause of death in women aged 40-50 years, accounting for approximately one in five of all deaths in this age group. More than 14,000 people die each year, and this incidence is rising. How to prevent it and prevent its recurrence has become a widely discussed topic. Meanwhile, data science has developed rapidly and is now

*Author's Email: ling.wang@ucdconnect.ie

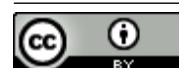

This work is licensed under a Creative Commons Attribution International 4.0 License.

IMIP '21, April 23-26, 2021, Tianjin, China

(C) 2021 Copyright held by the owner/author(s)

ACM ISBN 978-1-4503-9005-7/21/04.

https://doi.org/10.1145/3468945.3468965 widely used in all aspects of our lives, such as data visualization, and we identify the following trends. Topic about fashion, infographic generalization, mobile, social media, geolocation, data mashups, big data, globalization and scientific communication [3],various algorithms of machine learning, big data, including analytics, is a powerful tool that will be as useful in healthcare as in other industries [4]. Studies have shown that they play a role in the medical field of various diseases such as diabetes and eye diseases.

Therefore, the aim of this paper is to investigate two questions by analyzing some breast cancer case data: 1 . the random forest algorithm accurately predicts the occurrence of breast cancer using the given features. 2. features in the data have the greatest impact on the occurrence of breast cancer and thus better prevent the occurrence of breast cancer in the world.

\section{INITIAL VISUALIZATION OF DATA}

Data visualization tools are used by industry and academia to support decision making. Visualization is most useful in business analysis to monitor all activities comprehensively and also to make timely decisions. Here, we start with a data visualization analysis of all the data, which allows us to initially determine the correlation of each feature with the occurrence of breast cancer in preparation for the next more detailed analysis. As shown in the figure 1, we have clearly presented the whole data set through basic data visualization operations. We have 9 features and 1 result for this breast cancer dataset. They are age, menopause, tumor size, invnode, node-cyst, deg-malig, breast, breast-extremity, and irradiat. Subsequently, we digitized the attributes of all features in order to facilitate the calculation of correlation later (Figure 2). Then each factor is compared with the final results whether recurrence was observed by preliminary screening. From Figures 3, 4, and 5, we could obtain the following conclusions from observations:

- Age: the proportion of recurrence was relatively highest in the age group 30-39 years, lowest in the age group 20-29 years, lower in the age group 70 and above, and not much difference in the other age groups.

- Time of onset and menopause Tumor size: the probability of recurrence is highest in the age group of $30-34 \mathrm{~mm}$, and the probability of recurrence generally shows an increasing trend with the increase of tumor.

- Number of lymph nodes: the probability of recurrence basically shows an increasing trend with the increase of number.

- Radiotherapy experience: after radiotherapy, the probability of recurrence will be increased to a greater extent.

- Degree of malignancy of tumor: the higher the degree of malignancy, the higher the probability of recurrence. The higher the degree of malignancy, the greater the probability of recurrence.

Because the current analysis are only through the visualization of data and not through the real test. In the next stage, we will use 


\begin{tabular}{|c|c|c|c|c|c|c|c|c|c|c|}
\hline & class & age & menopause & tumor-size & inv-nodes & node-caps & deg-malig & breast & breast-quad & irradiat \\
\hline 0 & no-recurrence-events & $40-49$ & premeno & $20-24$ & $0-2$ & no & 2 & right & right_up & no \\
\hline 1 & no-recurrence-events & $40-49$ & premeno & $20-24$ & $0-2$ & no & 2 & left & left_low & no \\
\hline 2 & no-recurrence-events & $60-69$ & ge 40 & $15-19$ & $0-2$ & no & 2 & right & left_up & no \\
\hline 3 & no-recurrence-events & $40-49$ & premeno & $0-4$ & $0-2$ & no & 2 & right & right_low & no \\
\hline 4 & no-recurrence-events & $60-69$ & ge 40 & $15-19$ & $0-2$ & no & 2 & left & left_low & no \\
\hline
\end{tabular}

Figure 1: Initial feature table

\begin{tabular}{|c|c|c|c|c|c|c|c|c|c|}
\hline & & age & menopause & tumor-size & inv-nodes & node-caps & deg-malig & breast-quad & irradiat \\
\hline 0 & 1 & 2 & 0 & 3 & 0 & 0 & 2 & 5 & 1 \\
\hline 1 & 1 & 2 & 0 & 3 & 0 & 0 & 2 & 2 & 1 \\
\hline 2 & 1 & 4 & 2 & 2 & 0 & 0 & 2 & 3 & 1 \\
\hline 3 & 1 & 2 & 0 & 0 & 0 & 0 & 2 & 4 & 1 \\
\hline 4 & 1 & 4 & 2 & 2 & 0 & 0 & 2 & 2 & 1 \\
\hline
\end{tabular}

Figure 2: Digitally processed feature table
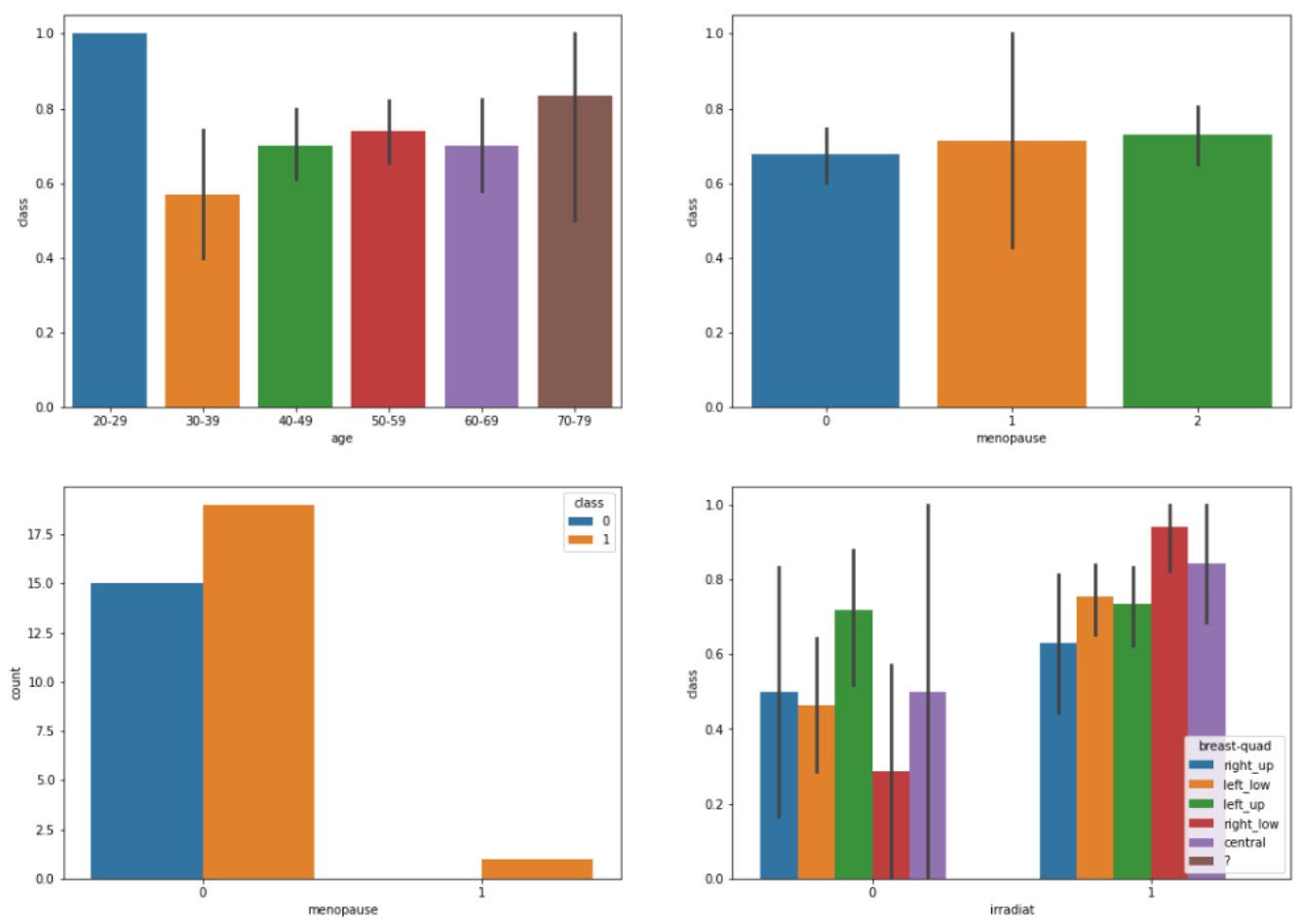

Figure 3: Chart showing the effect of age, menopause and other factors on recurrence rate

the random forest algorithm to verify the correlation of each feature with breast cancer recurrence and the accuracy of prediction. 

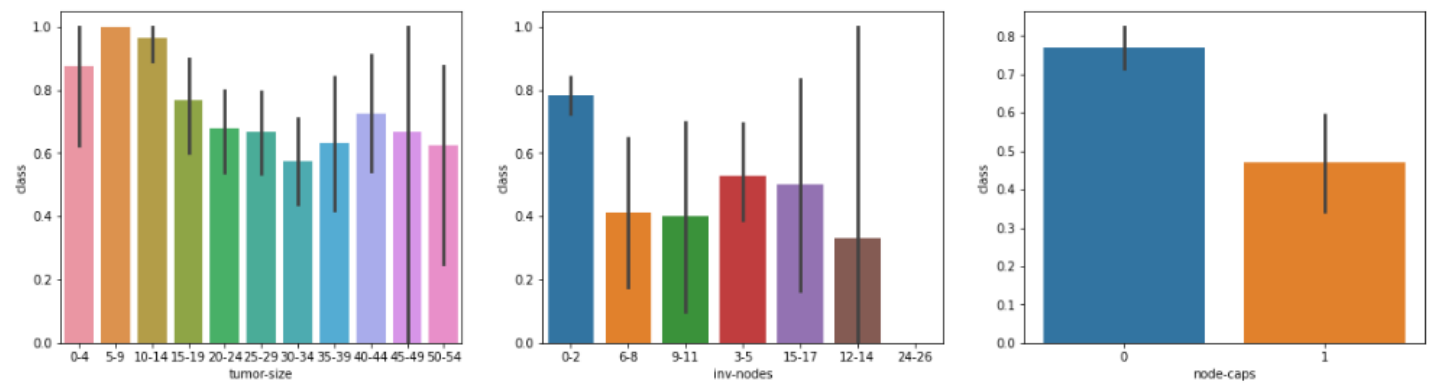

Figure 4: Diagram of the effect of tumor size, number of lymph node infections and other factors on recurrence rate

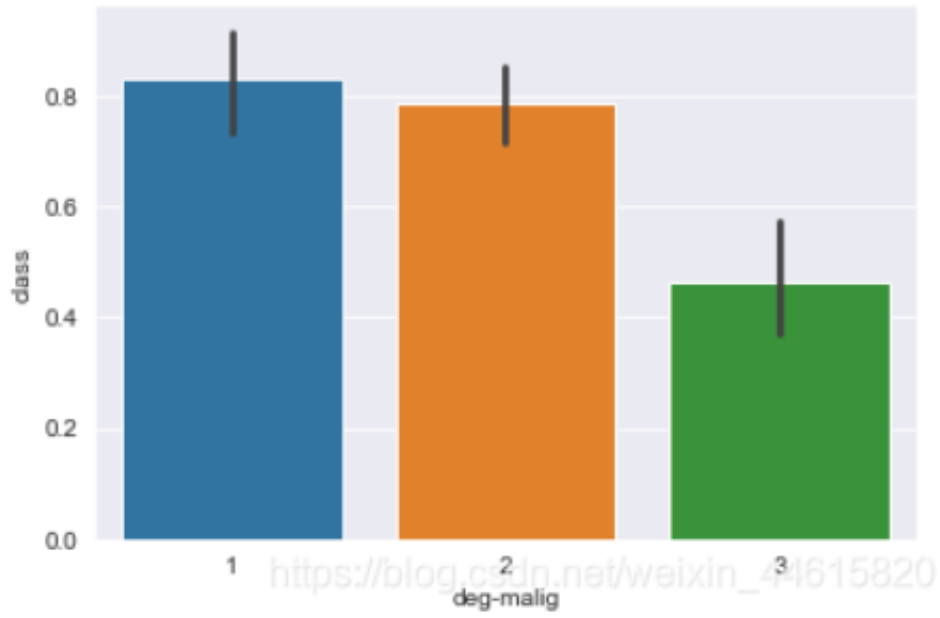

Figure 5: Diagram of the effect of tumor malignancy on recurrence rate

\section{PREDICTION OF DATA USING RANDOM FOREST ALGORITHM}

Random forest is a flexible and easy-to-use machine learning algorithm that brings good results in most cases even without hyperparameter tuning. It can be used for both classification and regression tasks. Unlike decision trees, more than decision trees, for regression problems, decision trees in random forests predict the value of Y (the output value). The final predicted value is calculated by averaging the predicted values of all decision trees in the random forest. As for the classification problem, each decision tree in the random forest predicts which classification the latest data belongs to.

Eventually, whichever classification is chosen the most will predict which classification the latest data belongs to. The random forest methodology has been successfully involved in a variety of practical problems, including data science hackathons for air quality prediction, cheminformatics, ecology, 3D object recognition, and bioinformatics, to name a few. Howard (Kaggle) and Bowles (Biomatica) in Howard and Bowles (2012) claim that decision trees of ensembles, often referred to as "random forests", are the most successful general-purpose algorithms of modern times [5]. The random forest algorithm can more efficiently and accurately help us to predict the correlation between individual features and breast cancer recurrence, and it is clearly the best choice for us as well. Training the classifier on a data set that is small and highly imbalanced will result in unpredictable results.

To overcome this issue, we used repeated random sub-sampling. Initially, we construct the testing data and the training data subsamples. For each disease, we train classifiers and test all of them on the same data set. The final labels of the testing data are computed using a majority voting scheme. To ensure the accuracy of the results and to prevent overfitting of the prediction results, we set numerical intervals for the algorithm to make predictions within the specified range, and the results showed that our prediction accuracy reached more than $80 \%$ in the test set. Based on this prediction result, we conducted further correlation analysis, and the results are shown in Figure 6. The degree of tumor malignancy, tumor size, and the number of lymph nodes are the main factors leading to breast cancer recurrence, while the remaining five factors also influenced the recurrence of breast cancer to different degrees.

\section{OTHER POTENTIAL FACTORS}

In fact, there are far more factors contributing to the development and recurrence of breast cancer than those described above. Weight, 


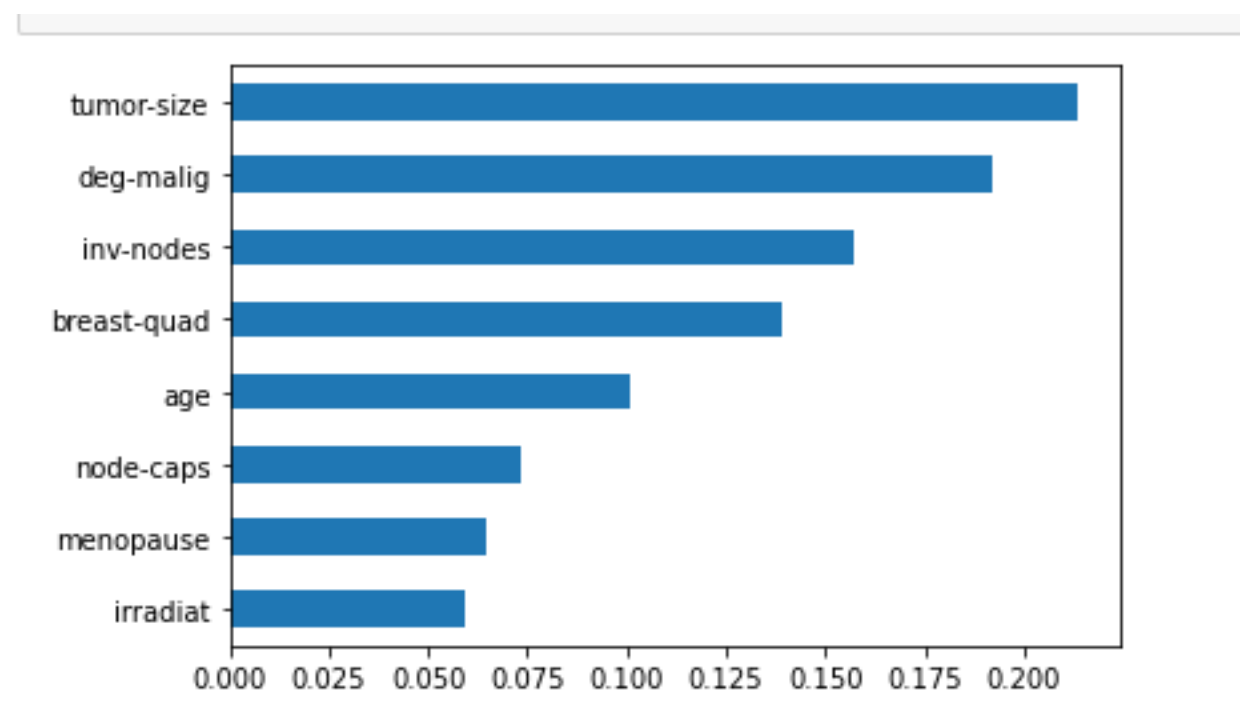

Figure 6: The correlation between various factors and breast cancer recurrence rate

family genetics are also thought to be strongly associated with the development and recurrence of breast cancer. 14,709 patients were recruited and treated at the Institut Curie (Paris) between 1981 and 1999. These patients were followed up for the first case of unilateral invasive breast cancer without distant metastases. Obesity was defined as a body mass index (BMI) higher than $30 \mathrm{~kg} / \mathrm{m} 2$ according to the recommendations of the World Health Organization. As a result, obese patients (8\%) showed more extended tumors at diagnosis, indicating a delay in the diagnosis of breast cancer. However, obesity appears to be a negative prognostic factor for multiple events in univariate and multifactorial survival analyses [6].

Family genetics is also a factor that should not be overlooked. A woman has twice or more times the risk of developing breast cancer if a first-degree relative (mother, sister, or daughter) develops the disease before age 50 . and the younger the relative who develops breast cancer, the greater her risk of developing breast cancer. risk. For example, a woman whose sister develops breast cancer between the ages of 30 and 39 has a $10 \%$ cumulative risk of developing the disease herself by age 65 , but that risk is only $5 \%$ (close to the population risk) if the sister is 50 years old. It was -54 at the time of diagnosis. If two first-degree relatives develop the disease, the risk increases four to six times. For example, women with two affected relatives, one under 50 years of age at diagnosis, have a $25 \%$ chance of developing breast cancer by age 65 [7]. All these factors demonstrate the variability and uncontrollability of breast cancer factors.

\section{DISCUSSION}

In the future, as algorithms improve, predictions will become more accurate and more characteristics and factors will be discovered such as weight, diet, genetics, daily routine, etc. People will also pay more attention to the prevention and recurrence of breast cancer to avoid them at the source. Preventive mastectomy is a means of reducing the risk of breast cancer. It is associated with a lower incidence of breast cancer in certain high-risk or highrisk populations [8]. Its use may be associated with reduced breast cancer mortality in BRCA mutation carriers. Lifestyle changes have also been advocated as a means of preventing breast cancer, mainly based on the association between these factors and lower risk. The feasibility of long-term lifestyle changes is controversial, but modest changes may be feasible for motivated women. A randomized trial of dietary fat reduction by the Women's Health Initiative found a small (9\%) reduction in breast cancer risk, which was statistically critical. Greater benefits were seen in more adherent women [9]. In the future, the diagnosis and treatment of breast cancer will continue to improve. Perhaps one day, when mortality rates are so low, the absolute number of lives saved through breast screening will be so low that screening may no longer be necessary.

\section{CONCLUSION}

This study and data analysis show that all the characteristics given in the data except the location of the tumor affect the recurrence of breast cancer to some extent. People experimentally demonstrate the accuracy of the random forest algorithm in predicting the recurrence of breast cancer, and also proved that data science can have a positive impact. In the future, more advanced equipment and research techniques will accelerate the study of breast cancer factors and improve the knowledge of breast cancer-related factors. The analysis of the breast cancer data set through data science is meaningful and valuable, and will have a positive impact and reference value for future analysis and research.

\section{ACKNOWLEDGMENTS}

First of all, I am very grateful to my advisor, Dr. Ta'Asan, for his careful guidance on my dissertation since winter break, which greatly improved my understanding of academic writing and taught 
me a lot of specific research skills; and to my teaching assistants who not only made summaries of our lessons every day, but also were able to answer my questions at the first time when I had the problems.

\section{REFERENCES}

[1] Waks AG, Winer EP. Breast Cancer Treatment: A Review. JAMA. 2019; doi:10.1001/jama.2018.19323, 321(3):288-300.

[2] Aparicio, M., \& Costa, C. J. (2015). Data visualization. Communication design quarterly review, 3(1), 7-11.

[3] David W. Bates, S. S. L. O.-M. A. S. a. G. E., 2014. Big Data In Health Care: Using Analytics To Identify And Manage High-Risk And High-Cost Patients. HEALTH
AFFAIRS, 33(7).

[4] Biau, G., Scornet, E. A random forest guided tour. TEST 25, 197-227 (2016). https: //doi.org/10.1007/s11749-016-0481-7

[5] Majed, B., Moreau, T., Senouci, K. et al. Is obesity an independent prognosis factor in woman breast cancer?. Breast Cancer Res Treat 111, 329-342 (2008). https://doi.org/10.1007/s10549-007-9785-3

[6] McPherson, K., Steel, C. M., \& Dixon, J. M. (2000). ABC of breast diseases. Breast cancer-epidemiology, risk factors, and genetics. BMJ (Clinical research ed.), 321(7261), 624-628. https://doi.org/10.1136/bmj.321.7261.624

[7] JNCI: Journal of the National Cancer Institute, Volume 103, Issue 18, 21 Septembe 2011, Pages 1397-1402, https://doi.org/10.1093/jnci/djr257

[8] Screening for breast cancer in England: past and future. Journal of Medical Screening.; 2006, doi:10.1258/096914106777589678, 13(2):59-61.

[9] Lostumbo L, Carbine NE, Wallace J. Prophylactic mastectomy for the prevention of breast cancer. Cochrane Database Syst Rev. 2010; 11:CD002748. 